\title{
Meningitis Caused by Methicillin Resistant - Staphylococcus aureus in Patient with Chronic Renal Insufficiency: A Case Report \\ M Jovanović ${ }^{1}$, M Dinić ${ }^{2}$, M Vrbić ${ }^{1}$, MP Floranović ${ }^{3 *}$, A Ranković ${ }^{1}$, LP Dragonjić ${ }^{1}$
}

\begin{abstract}
Meningitis caused by Staphylococcus aureus is a rare illnes with high mortality rate. It is associated with neurosurgery complications, and bacteremia arising from various inflamation foci. Predisposing factors are immunocompromised conditions. We report a case of methicillin resistent-Staphylococcus aureus meningitis in patient on chemodyalisis. Bacteremia originated from suppurative infection of right knee and shoulder. The patient made a full recovery after treatment with vancomycin and chloramphenicol. Regarding meningitis caused by $S$. aureus we emphasize the importance of locating and treating underlying focus of infection.
\end{abstract}

Keywords: Haemodialysis, meningitis, osteomyelitis, Staphylococcus aureus

From: ${ }^{1}$ Clinic for Infectious Diseases. University of Niš Clinics Centre, ${ }^{2}$ Institute for Public Health, ${ }^{3}$ Faculty of Medicine, Institute for Biomedical Research, University of Niš, Zoran Đinđić Boulevard 81, 18000 Niš, Serbia.

Correspondence: Dr M Potic Floranovic, Faculty of Medicine, Institute for Biomedical Research, University of Niš, Zoran Đinđić Boulevard 81, 18000 Niš, Serbia.

E-mail: milenapotic@yahoo.com 


\section{INTRODUCTION}

Staphylococcus aureus (S. aureus) is an uncommon cause of meningitis, accounting for 1-9\% of all bacterial meningitis (1). Mortality rates $(14 \%-77 \%)$ are higher than for other types of bacterial meningitis (2). Meningitis caused by methicillin - resistant S. aureus (MRSA) usually develops as a complication of neurosurgery, head trauma, or it can be acquired by haematogenous dissemination of focal infection (pneumonia, osteomyelitis, endocarditis, sinusitis, infected intravascular grafts). Predisposing factors for development of MRSA meningitis include: malignancy, diabetes mellitus, haemodialysis and other immunocompromised conditions (3-5). We describe a case of meningitis due to hematogenous dissemination of MRSA in non-neurosurgical patient with chronic renal insufficiency. This has been the first case of MRSA meningitis on our clinic in the last 20 years.

\section{CASE REPORT}

A 65 year old man was admitted to the clinic for infectious diseases with a 5-day history of severe occipital headache, and temperature of $37^{\circ} \mathrm{C}$. One day before admission he became agitated and confused. Patient was also on haemodialysis programme for the last two years, due to the chronic kidney failure.

On admission the patient was somnolent, agitated, adynamic, cahectic and afebrile. Blood pressure was $150 / 90 \mathrm{mmHg}$ and pulse 103 beats/min. Neurological examination revealed signs of meningeal irritation (stiff neck, Kerning's and Brudzinski's signs). A physical examination revealed a warm localised erythematous swelling in the region of the right knee and right shoulder. Initial laboratory results were as follows: elevated white blood cells (WBC) count of $23.5 \times 10^{3} / \mathrm{mmc}$ with $93.6 \%$ neutrophils and $3.7 \%$ lymphocytes; red 
blood cells: $3.95 \times 10^{6} / \mathrm{mmc}$; haemoglobin: $107 \mathrm{~g} / \mathrm{L}$, haematocrit 29.7 platelets: 185 , Creactive protein: $139.9 \mathrm{mg} / \mathrm{L}$; fibrinogen: $17.2 \mathrm{~g} / \mathrm{L}$; Urea $31 \mathrm{mmol} / \mathrm{L}$, creatinin: $562 \mathrm{mmol} / \mathrm{L}$. Other biochemical parameters were within normal range. On the admission day, lumbar puncture was performed. Cerebrospinal fluid (CSF) and blood sample was taken for culture. Laboratory analysis of CSF showed large number of polymorphonuclear cells, raised concentrations of proteins $(7.8 \mathrm{~g} / \mathrm{L})$ and decreased glucose concentration $(0.1 \mathrm{mmol} / \mathrm{L}$; simultaneous serum glucose $4.7 \mathrm{mmol} / \mathrm{L})$. Gram-stained smear of the CSF revealed polymorphonuclear leukocytes without organisms. Initial CSF was also inoculated onto Columbia agar, chocolate agar and MacConkey agar plates and tube of thioglycolate broth.

The initial antimicrobial therapy included rifampicin $300 \mathrm{mg} / 12 \mathrm{~h}$ and vancomycin $1.0 \mathrm{~g} / 24 \mathrm{~h}$. Four days after admission the patient's condition did not improve. Cultures from CFU and blood were positive for MRSA. Another lumbar puncture was performed, and cytological analysis showed once more pleocytosis $\left(\mathrm{WBC} 160 / \mathrm{mm}^{3}\right.$ ), raised concentrations of proteins $(7.25 \mathrm{~g} / \mathrm{L})$ and decreased glucose concentration $(0.2 \mathrm{mmol} / \mathrm{L}$; simultaneous serum glucose $4.4 \mathrm{mmol} / \mathrm{L}$ ). Computed tomography scan of the patient's head, sinuses and facial bones was performed. However, it revealed no evidence of bleeding, fracture or infarct. Initial physical examination suggested existence of the inflammation in the right knee and shoulder, $\mathrm{X}$ - rays of patient's genual joint and the glenohumeral joint area were taken. Knee $\mathrm{X}$-ray showed signs of irregular soft tissue formation around knee joint with change in bone density, and shoulder X-ray showed humeral head bone loss.

According to the susceptibility test the MRSA was susceptible only to vancomycin and chloramphenicol. Antibiotic therapy was modified: vancomycin $1.0 \mathrm{~g} / 24 \mathrm{~h}$ and chloramphenicol $1.0 \mathrm{~g} / 6 \mathrm{~h}$. Doses were adjusted according to the creatinine clearance.

Since both physical and radiological findings suggested there were infection foci in knee and shoulder joints, surgical consultation was required. On the sixth day, debridement of the right 
knee and shoulder was performed. Pyogenic content was removed and sent to microbiological and histopathological examination. Histopathology revealed bone necrosis and extensive inflammation, and culture grew methicillin resistant-S. aureus.

One day after therapy modification and surgical treatment, the condition of the patient started to improve. Two weeks after the treatment with vancomycin and chloramphenicol was initiated, lumbar puncture showed 14 lymphocytes and proteinorachia $1.09 \mathrm{~g} / \mathrm{L}$ which was interpreted within patients' age and previous inflammation. Antibiotic regiment lasted for three weeks. After that, control laboratory results were within normal range. Both control CSF and blood cultures were sterile, and patient was dismissed without sequelae.

\section{DISCUSSION}

Staphylococcal bacteraemia and disseminated abscesses are part of the Staphylococcus induced spectrum of diseases. S.aureus penetrates the blood brain barrier with difficulty, and it is a rear cause of meningitis. However, prolonged infections in immunocompromised individuals may promote bacteraemia and the development of meningitis. Host susceptibility, as with immune comprised individuals, plays a major role in hematogenous dissemination of S.aureus. (6) Methicillin resistance is associated with an increased risk for hematogenous complications such as infective endocarditis, septic arthritis, or osteomyelitis caused by hematogenous seeding from the infected site. (7). Haematogenous meningitis is associated with older age, community-acquired infection, underlying diseases, staphylococcal infection outside the central nervous system, and septic shock (8).

We describe an immunocompromised patient on haemodialysis with haematogenous dissemination of MRSA. We presume that the primary infection with $S$. aureus originated from the haemodialysis shunt, which is described in literature $(9,10)$. Patients on 
haemodialysis are at greater risk for bacterial infection, particularly S. aureus infection. The annual incidence of $S$. aureus bacteraemia in patients on haemodialysis ranges from 6 to $27 \%$ (9). Mechanisms of infection include contamination or inadequate disinfection of dialyzers during reprocessing, and contamination of bloodstream tubing by bacteria in expended priming saline or dialysate. (10). Catheter access is a primary risk factor for infection among patients on haemodialysis, but one in 12 patients receiving dialysis via arteriovenous fistula or synthetic or heterologous tissue graft develop S. aureus infection (9). Infection of the access site may be clinically silent or associated with only subtle clinical signs. In other cases, access site infection is presumed only when the patient develops metastatic foci of infection such as endocarditis, septic pulmonary emboli, septic arthritis, or osteomyelitis (11). In our case, prolonged methicillin-resistant $S$. aureus colonization of the haemodialysis access site probably caused osteomyelitis of the genual joint and the glenohumeral joint area. In Danish nationwide study, a significant number of the patients with $S$ aureus meningitis $(60 \%)$ had secondary focuses such as endocarditis (36\%) or osteomyelitis (16\%). Those conditions presented with non-specific and vague symptoms (2). This is consistent with our case, where chronic osteomyelitis and suppurative arthritis remained undetected at first. This was due to insufficient symptomatology caused by overall immunedefficiency of this patient. Therefore, the crucial moment in diagnosis and subsequently in therapy of our patient was identification and treatment of the inflammation foci.

Vancomycin is the antimicrobial agent most often used to treat MRSA infections. (12) In patients with chronic kidney failure it is necessary to adjust the dose in order to avoid toxicity. That can lead to treatment failure. However, there is no consensus on vancomycin dosing for patients with chronic kidney disease and with meningitis. (8) Our patient was initially treated with combination of vancomycin and rifampicin, which is a common clinical practice in many institutions. (13) Therapy modification according to the susceptibility of 
isolate was necessary since initial therapy was only partially adequate. High proportion of MRSA isolates in different areas of the world remain susceptible to chloramphenicol. Due to its myelotoxicity, and the lack of clinical experience, it is recommended to limit its use to situations where no alternative is available. (14).In our patient's case the combination of vancomycin and chloramphenicol has been effective.

In patients with staphylococcal meningitis and bacteraemia, there has to be a suspicion for osteomyelitis. The suggestive symptoms include pain and swelling, usually at the distal ends of long bones and in the vertebral bodies. Chronic infection can continue for months or even years, so this diagnosis must always be considered in any patient with staphylococcal bacteraemia and appropriate symptoms.

\section{CONCLUSION}

Early diagnosis of the central nervous system infections is a precondition of their successful treatment. Regarding meningitis caused by $S$. aureus we emphasize the importance of locating and treating underlying focus of infection (osteomyelitis, haemodialysis shunts, tissue abscess, cellulitis). Initiation of appropriate antibiotic treatment must not be delayed. Surgical intervention and bone-penetrating, CNS penetrating, antibiotic therapy should close the vicious circle of bacteraemia. 


\section{REFERENCES}

1. Schlesinger LS, Ross SC, Schaber DR. Staphylococcus aureus meningitis: a broadbased epidemiologic study. Medicine 1987; 66 (Suppl 2): 148-56.

2. Pedersen M, Benfield TL, Skinhoej P, Jensen AG. Haematogenous Staphylococus aureus meningitis. A 10-year nationwide study of 96 consecutive cases. BMC Infectious Diseases 2006; 6-49.

3. Hussein AS, Shafran SD. Acute bacterial meningitis in adults. A 12-year review. Medicine 2000; 79:360-8.

4. Logigan C, Mihalache D, Dorneanu O, Turcu T. Analysis of 62 cases of nosocomial staphyloccocal meningits admited to the Iasi Hospital of Infectious Diseases over a period of 21 years. Rev Med Chir Soc Med Nat Iasi. 2010; 114 (Suppl 1): 106-10.

5. Dylewski J, Martel G, A. case of spontaneous methicillinresistant Staphylococcus aureus meningitis in a health care worker. Can J Infect Dis Med Microbiol 2004; 15(6):336-338.

6. Jensen AG, Espersen F, Skinhoj P, Rosdahl VT, Frimodt-Moller N. Staphylococcus aureus meningitis .A review of 104 nationwide, consecutive cases. Arch Intern Med 1993; 153(Suppl 16):1902-8.

7. Fowler VG, Justice A, Moore C, Benjamin DK, Woods CW, Campbell S. Risk Factors For Hematogenous Complications of Intravascular Catheter-Associated Staphylococcus aureus Bacteremia. Clin Infect Dis.2005 1; 40 (Suppl 5):695-703.

8. Wen-Chi Huang, Chen-Hsiang Lee, Jien-Wei Liu. Clinical Characteristics and Risk Factors for Mortality in Patients with Meningitis Caused by Staphylococcus aureus and Vancomycin Minimal Inhibitory Concentrations against These Isolates. J Microbiol Immunol Infect 2010; 43 (Suppl 6):470-477 
9. Li Y, Friedman JY, O’Neal BF, Hohenboken MJ, Griffiths RI, Stryjewski ME, et al. Outcomes of Staphylococcus aureus infection in haemodialysis-dependent patients. Clin J Am Soc Nephrol 2009; 4: 428-34.

10. Fujimoto C, Yazawa S, Matsuoka F, Ishihara A, Hayami M, Kawasaki S. Bacterial meningoencephalitis in patients undergoing chronic hemodialysis: two case reports. Clin Neurol Neurosurg. 2002; 104(Suppl 1):64-8.

11. Lentino JR, Badour LM, Wray M, Wong ES, Yu VL. Staphylococcus aureus and other bacteremias in hemodyalisis patients: Antibiotic therapy and surgical removal of access site. Infection 2000; 28(Suppl 6): 355-60.

12. Rybak, $\mathrm{M}$ et al. Therapeutic monitoring of vancomycin in adult patients: a consensus review of the American Society of Health-System Pharmacists, the Infectious Diseases Society of America, and the Society of Infectious Diseases Pharmacists. Am J Health-Syst Pharm 2009; 66 (Suppl 1): 82-98.

13. Watanakunakorn C, Guerriero JC. Interaction between vancomycin and rifampin against Staphylococcus aureus Antimicrob Agents Chemother, 19 (1981), pp. 10891091.

14. Bouza E. New therapeutic choices for infections caused by methicillin-resistant Staphylococcus aureus. Clin Microbiol Infect. 2009; 15(Suppl 7):44-52. 